(2) Open Access Full Text Article

\title{
Reversal of echocardiographic right-sided heart pathology in a dog with severe pulmonary hypertension: a case report
}

This article was published in the following Dove Press journal:

Veterinary Medicine: Research and Reports

3 June 2015

Number of times this article has been viewed

\author{
Peggy McMahon' \\ Carley Saelinger ${ }^{2}$ \\ 'Emergency and Critical Care \\ Department, ${ }^{2}$ Cardiology Department, \\ Animal Specialty and Emergency \\ Center, Los Angeles, CA, USA
}

\begin{abstract}
Pathologic right-sided heart changes are a common echocardiographic finding in patients with pulmonary hypertension (PH). Canines with $\mathrm{PH}$ may have right heart pathology documented via echocardiographic color Doppler interrogation including tricuspid valve regurgitation, pulmonic valve insufficiency, elevated pulmonary arterial systolic pressure, elevated pulmonary arterial diastolic pressure, and alterations in ejection profiles. Two-dimensional echocardiographic findings may include right ventricular hypertrophy, interventricular septal flattening, paradoxical interventricular septal motion, pulmonary artery dilation, and potentially abnormal left heart dimensions. In veterinary medicine, much confidence is given to the measurement of pulmonary arterial systolic pressure estimated from tricuspid valve regurgitation to grade the severity of $\mathrm{PH}$ and monitor its improvement with little emphasis placed on the integration of two-dimensional echocardiographic right and left heart pathology in conjunction with Doppler findings. To the authors' knowledge, marked improvement and/or resolution of echocardiographic-documented right heart pathology have not been previously reported in the veterinary literature. This case report documents profound echocardiographic improvement of right-sided heart disease in a dog with severe $\mathrm{PH}$.
\end{abstract}

Keywords: canine, pulmonary hypertension, tricuspid valve regurgitation, right heart hypertrophy, sildenafil

\section{Introduction}

Pulmonary hypertension (PH) is a global term for a complex hemodynamic condition leading to a progressive increase in pulmonary vascular pressures. $\mathrm{PH}$ is defined as an estimated pulmonary arterial systolic pressure (PASP) greater than $30 \mathrm{mmHg}$ or pulmonary arterial diastolic pressure greater than $19 \mathrm{mmHg}$. It can be classified as increased precapillary resistance (pulmonary arterial hypertension, resulting from abnormalities on the arterial side of the pulmonary vascular system) or increased postcapillary resistance (pulmonary venous hypertension, resulting from left-sided heart disease leading to pulmonary capillary hypertension). In dogs, PH may be a consequence of various disease processes, including an idiopathic cause, primary cardiac disease, intra- and extracardiac shunts, heartworm disease, respiratory disease, and thromboembolic disease. Clinical signs associated with PH include tachypnea, cough, respiratory distress, weakness, fatigue, exercise intolerance, syncope, and right-sided congestive heart failure. ${ }^{1-3}$

In health, the right ventricle (RV) and pulmonary circulation function as a coupled unit. In diseased states, the normally thin-walled, distensible RV becomes incapable of completely matching, or coupling, its contractile performance to
Correspondence: Peggy McMahon

Emergency and Critical Care

Department, Animal Specialty and

Emergency Center, 1535 South Sepulveda

Boulevard, Los Angeles, CA 90025, USA

$\mathrm{Tel}+\mid 310473$ |56|

Fax + I 3104798976

Email peggymcmahondvm@gmail.com 
increased pulmonary vascular resistance (PVR), resulting in right ventricular-pulmonary artery (PA) uncoupling. Two-dimensional echocardiographic findings including RV hypertrophy (eccentric and concentric), right atrial hypertrophy, interventricular septal flattening, paradoxical interventricular septal motion, and PA dilation support the diagnosis of PH. ${ }^{4}$ Eccentric and/or concentric RV hypertrophy may occur with acute and chronic RV pressure overload. The degree to which they occur can help indicate chronicity and underlying pathology. ${ }^{1,5-7}$ The causes of $\mathrm{PH}$ can be narrowed down from a noninvasive, thorough echocardiographic interpretation.

Echocardiography provides the most informative and least invasive means of evaluating cardiac structure and function in the veterinary clinical setting when $\mathrm{PH}$ is suspected. Objective maximum velocity measurements of tricuspid valve regurgitation and pulmonic valve insufficiency are the most commonly used criteria to make a diagnosis of $\mathrm{PH}$ in veterinary medicine. Pairing echocardiographic Doppler interrogation findings with subjective assessment of right- and left-sided cardiac structures, measurements of right ventricular systolic function, and right ventricular ejection profiles can provide a more comprehensive evaluation and perhaps a more specific cause for the PH present.

This case is the first clinical report that describes marked clinical, two-dimensional, and color Doppler echocardiographic improvement in a dog treated for $\mathrm{PH}$. Other veterinary studies have shown positive responses to treatments, including resolution of syncopal events, improved exercise tolerance, decreased PA pressure, ${ }^{3,8}$ decreased pressure gradients through the tricuspid valve, ${ }^{9}$ and increased quality of life despite a lack of significant change in other variables. ${ }^{5}$ Much focus is also given to observing reduction in peak tricuspid regurgitant flow velocity (PTRFV) as estimated via continuous wave interrogation of tricuspid valve regurgitation using the simplified Bernoulli equation. However, this value alone is incomplete in assessing the severity and potential outcome of disease. ${ }^{4}$ PTRFV does not reliably decrease despite improvement in clinical signs. ${ }^{4,5,10}$ In human medicine, PASP is a poor measure of RV afterload, explaining why PA pressure is also a poor predictor of clinical RV failure and prognosis. PH may still be considered severe if persistent pathological changes in RV shape and size, RV systolic dysfunction, and abnormal interventricular septal motion are present despite insufficient tricuspid or pulmonic valve regurgitation and elevated PA pressures. ${ }^{4}$

\section{Case report}

A 2-year-old castrated male Chihuahua was presented to the Cardiology Service for initial evaluation of labored breathing. Pertinent medical history includes a diagnosis of rabies vaccine-induced ischemic dermatopathy via dermal histopathology by a board-certified veterinary dermatologist 1 year prior to presentation. The condition was treated with immunomodulatory, antibiotic, and antifungal therapy. Ten months after the treatment was instituted, additional dermal histopathology showed scarring with no persistent evidence of ischemic dermatopathy. All therapies were discontinued.

The patient was presented to the Cardiology Service with a several-day history of increased respiratory rate, decreased appetite, and lethargy. Upon initial evaluation, the patient was alert and responsive. Vitals included a heart rate of 140 beats per minute, respiratory rate of 60 breaths per minute, and body temperature of $36.67^{\circ} \mathrm{C}\left(98^{\circ} \mathrm{F}\right)$. Physical examination revealed a new-grade V/VI right apical systolic heart murmur, mild tachypnea with mild respiratory effort, normal bronchovesicular sounds, and severe generalized multifocal alopecia with scarring. Thoracic radiographs revealed generalized cardiomegaly and main PA enlargement evidenced by a bulge at the 1-2 o'clock position on the ventrodorsal projection. There was a moderate, diffuse unstructured interstitial pulmonary pattern, with heavier infiltrates evenly distributed throughout the right hemithorax.

Echocardiography revealed a mildly thickened mitral valve with mild valve regurgitation, small left ventricle (LV) internal dimensions in diastole and systole, and normal left atrium (LA) size. Color Doppler interrogation of the LA revealed diastolic inflow turbulence from right PA compression. Severe eccentric hypertrophy of the RV, mild concentric hypertrophy of the RV, severe enlargement of the right atrium (RA), paradoxical interventricular septal motion, systolic interventricular septal flattening, and reduced LV internal diameters were noted (Figure 1 and Table 1). The tricuspid valve was mildly thickened with severe tricuspid valve regurgitation present (Figures 2 and 3 ). The right ventricular-to-right atrial pressure gradient at the end of systole was estimated from PTRFV using continuous wave Doppler and the modified Bernoulli equation at $5.68 \mathrm{~m} / \mathrm{s}$, corresponding to an estimated right ventricular end-systolic pressure (RVESP) of $129 \mathrm{mmHg}$ (approximates PASP in the absence of RV outflow obstruction) (Figure 2 and Table 2). The pulmonic valve appeared structurally normal. Mild pulmonic valve insufficiency was present, and an interrogation with continuous wave Doppler revealed an elevated end 

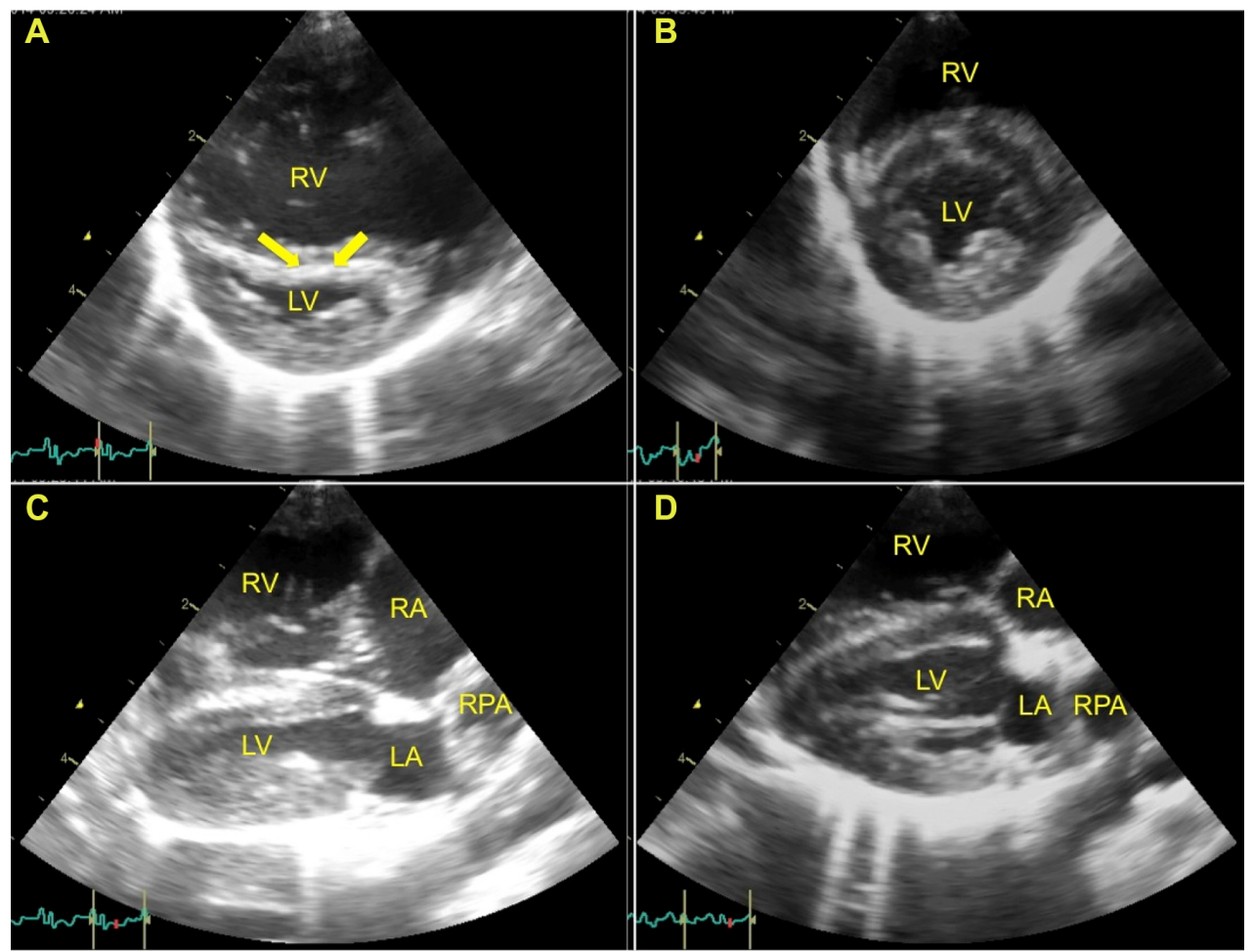

Figure I Right parasternal short-axis echocardiographic images of LV and RV, and long-axis four-chamber 2-D echocardiographic images.

Notes: (A and B) Right parasternal short-axis echocardiographic images showing a cross section of the LV and RV at the level of papillary muscles. (A) Severe eccentric RV hypertrophy, mild RV concentric hypertrophy, and interventricular septal flattening during systole are noted (arrows). The LV is small and underfilled, and pseudo LV concentric hypertrophy is present. (B) Four weeks after sildenafil administration. Mild concentric RV hypertrophy remains, eccentric RV hypertrophy is now mild, there is normal septal motion, and the LV is normal in dimensions. (C and D) Right parasternal long-axis four-chamber 2-D echocardiographic images. (C) Severe eccentric RV hypertrophy, mild RV concentric hypertrophy, severe RA enlargement, and moderate RPA dilation are noted. The LV is small and underfilled, pseudo LV concentric hypertrophy is present, and the LA appears normal in size. (D) Four weeks after sildenafil administration. Mild concentric RV hypertrophy remains, eccentric RV hypertrophy is now mild, and the LV subjectively appears more volume loaded. The LA is normal in size. The RA is now borderline enlarged, and the RPA remains moderately dilated. Abbreviations: LA, left atrium; LV, left ventricle; RA, right atrium; RPA, right pulmonary artery; RV, right ventricle; 2-D, two-dimensional.

pulmonary arterial diastolic pressure of $43 \mathrm{mmHg}(3.36 \mathrm{~m} / \mathrm{s})$. The main PA and branches were moderately to severely dilated. Right ventricular outflow tract (RVOT) and PA tracing showed a laminar flow of $1 \mathrm{~m} / \mathrm{s}$ with mid-systolic notching, suggesting increased PVR (Figure 4 and Table 2).

Table I Echocardiographic two-dimensional parameters

\begin{tabular}{|c|c|c|c|c|}
\hline \multirow[t]{2}{*}{ Variable } & \multicolumn{2}{|c|}{ Pretherapy } & \multicolumn{2}{|c|}{$\begin{array}{l}\text { I month } \\
\text { posttherapy }\end{array}$} \\
\hline & Diastole & Systole & Diastole & Systole \\
\hline $\begin{array}{l}\text { Interventricular septum } \\
(\mathrm{mm})\end{array}$ & 4.23 & 5.70 & 3.87 & 5.89 \\
\hline $\begin{array}{l}\text { Left ventricular internal } \\
\text { diameter }(\mathrm{mm})\end{array}$ & 8.35 & 3.35 & $10.7 \mid$ & 5.15 \\
\hline $\begin{array}{l}\text { Left ventricular } \\
\text { posterior wall (mm) }\end{array}$ & 5.13 & 6.46 & 4.36 & 5.15 \\
\hline Fractional shortening (\%) & 59.86 & & 51.91 & \\
\hline Left atrium (mm) & 11.19 & & 12.92 & \\
\hline Aorta (mm) & 8.40 & & 8.71 & \\
\hline Left atrium: aorta & 1.33 & & 1.48 & \\
\hline Pulmonary artery (mm) & 10.08 & & 10.42 & \\
\hline Pulmonary artery: aorta & 1.20 & & 1.20 & \\
\hline
\end{tabular}

The RVOT acceleration time was shortened and the RVOT acceleration time to ejection time was decreased (Table 2). There was no evidence of pericardial or pleural effusion. A bubble study with agitated saline was negative for intra- and extrathoracic shunts. A lead II electrocardiogram revealed a normal sinus rhythm to sinus tachycardia throughout the study. Findings were consistent with severe PH from pulmonary vascular disease (PVD), a precapillary cause of $\mathrm{PH}$.

Additional testing was performed in an effort to elucidate an underlying cause for severe PH. Abnormal hematological findings included a mild regenerative anemia characterized by a reticulocytosis, neutrophilic, monocytic leukocytosis with a left shift, and a moderate thrombocytopenia. Abnormal serum chemistry findings included a mildly increased alkaline phosphatase, mildly increased alanine aminotransferase, mild hypoproteinemia, and mild hypoalbuminemia (Table 3). A thyroid panel and urinalysis were normal. A thromboelastogram revealed normal coagulation parameters. Heartworm and tick-borne disease testing, including polymerase chain reaction assays, were negative. An antinuclear antigen assay 


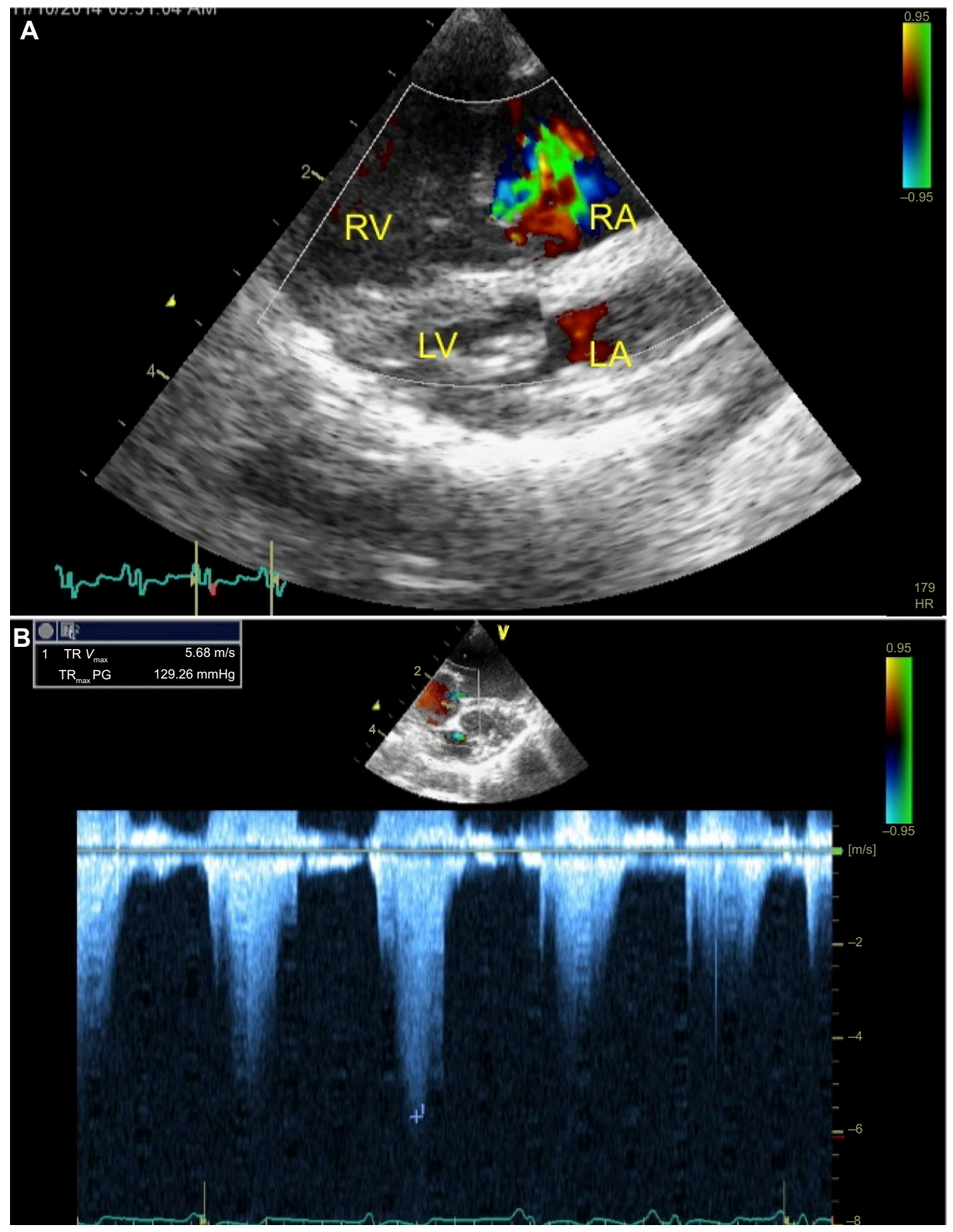

Figure 2 Right parasternal long- and short-axis 2-D echocardiographic images with color Doppler and continuous wave color Doppler at the level of the tricuspid valve, respectively.

Notes: (A) Right parasternal long-axis four-chamber 2-D echocardiographic image with color Doppler at the level of the tricuspid valve. Moderate-to-severe TR, severe eccentric RV hypertrophy, mild RV concentric hypertrophy, and severe RA enlargement are noted. The LV is small and underfilled, pseudo LV concentric hypertrophy is present, and the LA is normal in size. (B) Right parasternal short-axis 2-D echocardiographic image with continuous wave color Doppler at the level of the tricuspid valve. TR peak velocity is measured and RVESP is estimated via the modified Bernoulli's equation at $129 \mathrm{mmHg}$.

Abbreviations: LA, left atrium; LV, left ventricle; RA, right atrium; RV, right ventricle; RVESP, right ventricular end-systolic pressure; TR, tricuspid valve regurgitation; TR $V_{\text {max }}$, maximum TR peak velocity; $T R_{\max }$ PG, maximum TR pressure gradient; $V$, velocity; 2-D, two-dimensional.

was negative. An abdominal ultrasound revealed caudal vena cava and hepatic vein distension, a small amount of anechoic peritoneal effusion, and a hypoechoic, mildly enlarged pancreas, thought to be a variation of normal in this patient. The patient was discharged with administration of sildenafil $2.40 \mathrm{mg} / \mathrm{kg}$ every 12 hours.

The patient was reevaluated by the Cardiology Service 2 days after his initial visit due to new coughing and worsening of labored breathing at home. Physical examination revealed a static heart murmur, diffusely increased bronchovesicular sounds, and soft crackles bilaterally. Compared to the previous visit's radiographs, there was worsening of the unstructured interstitial pulmonary pattern. The patient was admitted to the hospital, placed in environmental oxygen at $40 \%$ for several hours, and sildenafil administration was increased in frequency to $2.40 \mathrm{mg} / \mathrm{kg}$ orally every 8 hours. By the 

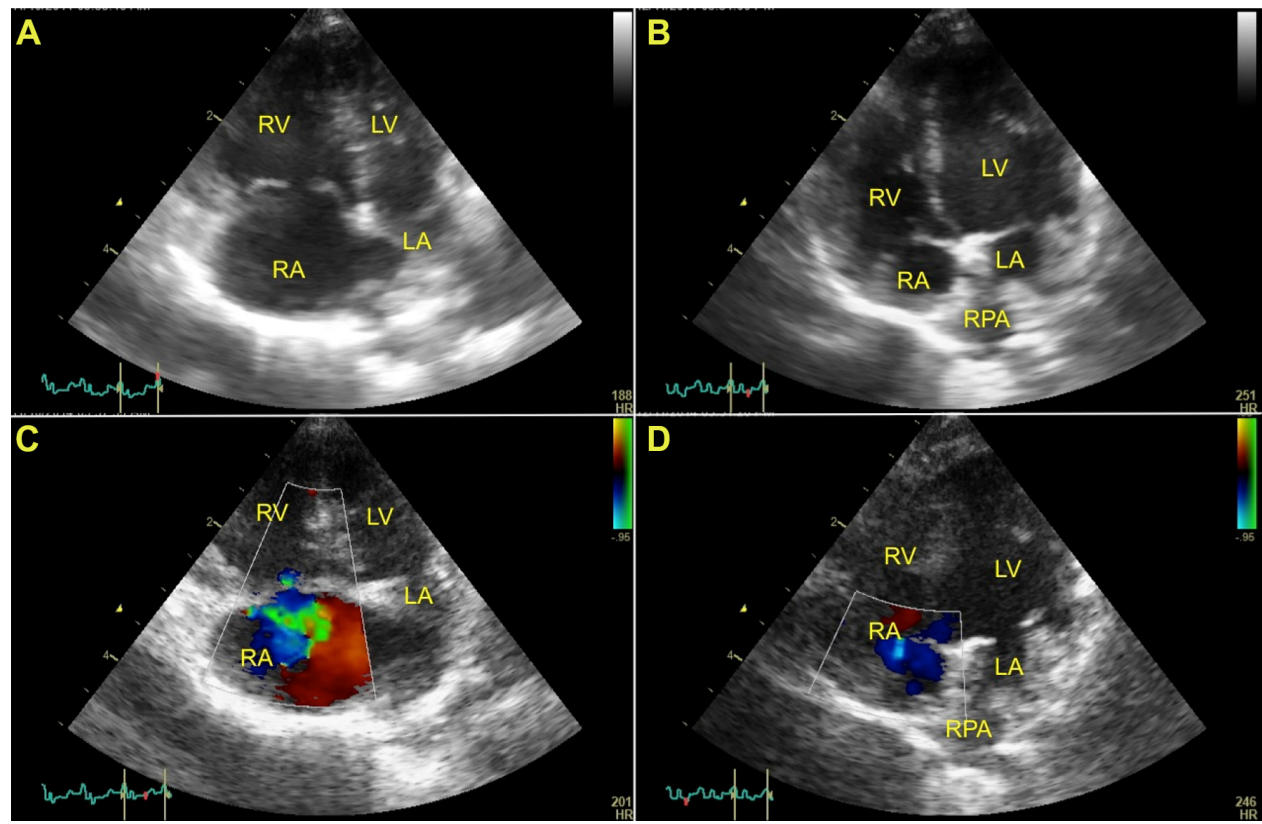

Figure 3 Left parasternal apical four-chamber 2-D echocardiographic images.

Notes: (A and B) Left parasternal apical four-chamber 2-D echocardiographic images. (A) Severe eccentric RV hypertrophy, mild RV concentric hypertrophy, and severe RA enlargement are noted. The LV is small and underfilled, pseudo LV concentric hypertrophy is present, and the LA is normal in size. The RPA is moderately dilated. (B) Four weeks after sildenafil therapy. Mild RV concentric hypertrophy persists, mild eccentric RV hypertrophy is now present, the RA is now borderline enlarged, the RPA remains moderately dilated, and the left heart appears more preload appropriate in size. (C and D) Left parasternal apical four-chamber 2-D echocardiographic images with color Doppler at the level of the tricuspid valve. (C) Moderate-to-severe tricuspid valve regurgitation, severe eccentric RV hypertrophy, mild RV concentric hypertrophy, severe RA enlargement, and moderate RPA dilation are noted. The LV is small and underfilled, pseudo LV concentric hypertrophy is present, and the LA appears small. (D) Four weeks after sildenafil administration. Tricuspid valve regurgitation is now trace, mild concentric RV hypertrophy remains, eccentric RV hypertrophy is now mild, the RA is now borderline enlarged, the RPA remains moderately dilated, and the LV and LA subjectively appear more volume loaded.

Abbreviations: LA, left atrium; LV, left ventricle; RA, right atrium; RPA, right pulmonary artery; RV, right ventricle; 2-D, two-dimensional.

following morning, the patient was breathing with only mild respiratory effort in room air. An echocardiogram revealed a moderate improvement in RVESP to $83 \mathrm{mmHg}$ and a subjective decrease in right heart size. The patient was discharged from the hospital with sildenafil as described earlier.

Table 2 Echocardiographic Doppler parameters

\begin{tabular}{lll}
\hline Variable & Pretherapy & $\begin{array}{l}\text { I month } \\
\text { posttherapy }\end{array}$ \\
\hline $\begin{array}{l}\text { Tricuspid regurgitant flow peak } \\
\text { velocity }(\mathrm{m} / \mathrm{s})\end{array}$ & 5.68 & $\begin{array}{l}\text { Insufficient } \\
\text { regurgitant jet } \\
\text { Insufficient } \\
\text { regurgitant jet }\end{array}$ \\
$\begin{array}{l}\text { Right ventricular end-systolic } \\
\text { pressure }(\mathrm{mmHg})\end{array}$ & 129.0 & $\begin{array}{l}\text { Insufficient } \\
\text { regurgitant jet }\end{array}$ \\
$\begin{array}{l}\text { Pulmonary arterial diastolic } \\
\text { flow velocity (m/s) }\end{array}$ & 3.36 & $\begin{array}{l}\text { Insufficient } \\
\text { regurgitant jet }\end{array}$ \\
$\begin{array}{l}\text { Pulmonary arterial diastolic } \\
\text { pressure (mmHg) }\end{array}$ & 43.0 & 0.9 \\
$\begin{array}{l}\text { Right ventricular outflow tract } \\
\text { maximum velocity (m/s) }\end{array}$ & 1.0 & 40.4 \\
$\begin{array}{l}\text { Right ventricular outflow tract } \\
\text { acceleration time (ms) }\end{array}$ & 20.2 & 89.99 \\
$\begin{array}{l}\text { Right ventricular outflow } \\
\text { ejection time (ms) }\end{array}$ & 119.38 & 0.45 \\
$\begin{array}{l}\text { Right ventricular outflow } \\
\text { acceleration time/ejection time } \\
\text { Left ventricular outflow tract } \\
\text { maximum velocity (m/s) }\end{array}$ & 0.17 & 0.93 \\
\hline
\end{tabular}

Approximately 1 month later, the patient was presented for a routine recheck examination. He was reported to be doing very well at home with normal activity, normal appetite, and eupnea. Physical examination revealed resolution of the right-sided heart murmur, a normal respiratory pattern, and normal bronchovesicular sounds. Thoracic radiographs revealed continued cardiomegaly and main PA enlargement, with improvement to a mild, diffuse unstructured interstitial pulmonary pattern. An echocardiogram revealed only trace tricuspid valve regurgitation (unable to estimate RVESP from trivial regurgitation present) and resolution of pulmonic valve insufficiency. Additionally, there were marked improvements in right heart findings, including only mild RV eccentric and concentric hypertrophy, borderline RA enlargement, normal RV ejection profiles, improved RVOT acceleration time and acceleration time/ejection time, normal interventricular septal motion, and a normal LV dimensions (Figures 1-4; Tables 1 and 2).

\section{Discussion}

The cause of this patient's PH is most consistent with PVD due to lack of significant changes to the left heart, increased PVR, abnormal PA compliance (notched RV ejections profiles), 


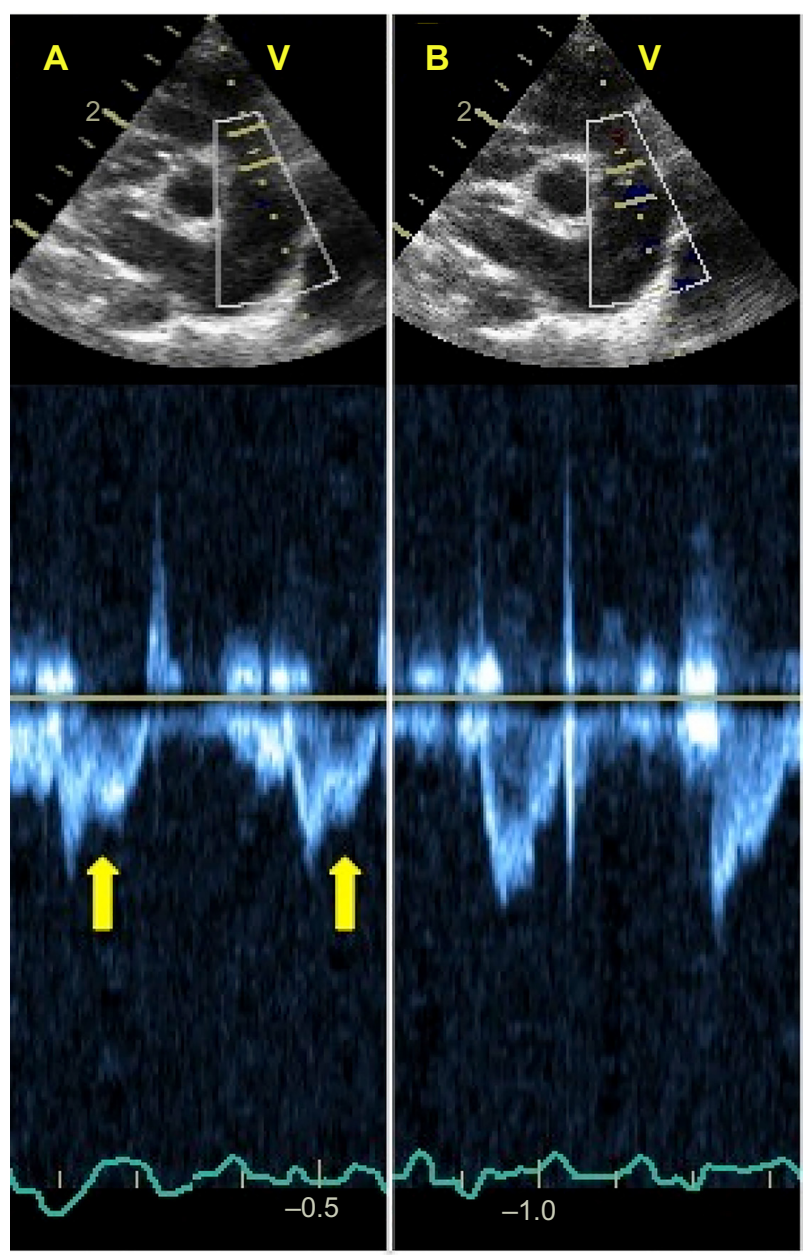

Figure 4 Right parasternal short-axis 2-D echocardiographic images with interrogation of the RVOT ejection envelopes/velocities with pulsed wave color Doppler.

Notes: (A) Mid-systolic notching (arrows) is noted in RVOT ejection envelopes. Moderate dilation of the main, right, and left pulmonary arteries is noted. (B) Four weeks after sildenafil administration. RVOT ejection profiles are normal in shape and laminar. Moderate dilation of the main, right, and left pulmonary arteries persists. Abbreviations: RVOT, right ventricular outflow tract; $V$, velocity; 2-D, twodimensional.

shortened RVOT acceleration time and decreased acceleration time/ejection time, alterations of RV systolic function (decreased longitudinal shortening of the RV free wall), increased RV size and changed morphology, and alterations in interventricular motion (systolic and diastolic paradoxical motion). These echocardiographic changes are hallmarks of those noted with right ventricular-PA uncoupling. However, conductance catheterization represents the gold standard method for evaluating ventriculoarterial coupling via measurements of ventricular and arterial elastance. ${ }^{4}$

As with many cases of $\mathrm{PH}$, the underlying cause of this patient's PH was not definitively determined. This patient suffered from chronic, severe ischemic dermal disease. Ischemic dermatopathy is believed to be the result of vascular abnormalities such as vasculopathy and
Table 3 Hematological and biochemical abnormalities

\begin{tabular}{lll}
\hline Parameter & Value & Reference interval \\
\hline Hematocrit $(\%)$ & 26.3 & $38.3-56.5$ \\
Reticulocyte $(\mathrm{K} / \mu \mathrm{L})$ & 132 & $10-110$ \\
WBC $(\mathrm{K} / \mu \mathrm{L})$ & 23.7 & $4.9-17.6$ \\
Neutrophil $(\mu \mathrm{L})$ & 16,116 & $2,940-12,670$ \\
Band $(\mu \mathrm{L})$ & 474 & $0-170$ \\
Monocyte $(\mu \mathrm{L})$ & 2,370 & $130-1,150$ \\
Platelet $(\mathrm{K} / \mu \mathrm{L})$ & 72 & $143-488$ \\
ALP $(\mathrm{U} / \mathrm{L})$ & 380 & $5-260$ \\
ALT $(\mathrm{U} / \mathrm{L})$ & 175 & $18-121$ \\
Total protein $(\mathrm{g} / \mathrm{dL})$ & 5.1 & $5.5-7.5$ \\
Albumin $(\mathrm{g} / \mathrm{dL})$ & 2.3 & $2.7-3.9$ \\
\hline
\end{tabular}

Abbreviations: ALP, alkaline phosphatase; ALT, alanine aminotransferase; WBC, white blood cell.

vasculitis from immunologically induced structural defects in microvasculature. ${ }^{11}$ In people, interstitial lung disease is a common respiratory manifestation of similar ischemic connective tissue disease, and it has been associated with $\mathrm{PH} .{ }^{12,13}$ To date, $\mathrm{PH}$ as a manifestation of ischemic connective tissue disease has not been reported in dogs. Although autoimmune disease is a plausible cause for this patient's $\mathrm{PH}$, other forms of chronic respiratory disease, idiopathic $\mathrm{PH}$, and thromboembolic disease remain possibilities.

Regardless of the cause of $\mathrm{PH}$, adaptations of the right heart secondary to increased afterload occur through mechanisms of hypertrophy and dilation. In the normal heart, the RV adapts to an increase in load with an increase in contractility. Compared to the LV, the RV free wall is much thinner and has greater compliance, making it more sensitive to increased afterload. In patients with $\mathrm{PH}$, stretching of the RV wall leads to adaptive remodeling (concentric hypertrophy) due to increased protein synthesis and increased cardiomyocyte size through the addition of sarcomeres. ${ }^{14}$ This adaptation cannot be maintained in the face of sustained pressure overload, and there is eventually a transition to dilation (eccentric hypertrophy). The specific mechanisms underlying the transition from hypertrophy to dilation in the right ventricular failure secondary to $\mathrm{PH}$ remain unclear. The degree of RV concentric hypertrophy versus eccentric hypertrophy can also help to elucidate the chronicity and possible causes of PH. Severe concentric RV hypertrophy is seen more with chronic disease, whereas severe eccentric RV hypertrophy, as seen in this patient, is typically noted in cases of acute RV dilation. ${ }^{4} \mathrm{RV}$ dilation causes an increase in myocardial oxygen demand while decreasing right-sided heart perfusion, and finally decreasing function.

There is no universally accepted consensus on the optimal method of assessing RV function and structure, in 
people and animals. In veterinary medicine, two-dimensional echocardiography can be supportive evidence of changes associated with PH and is often underused in helping to distinguish PH from PVD versus pulmonary venous hypertension and in monitoring response to treatment. In this patient, significant right-sided cardiac pathology associated with $\mathrm{PH}$ from primary PVD included profound RV eccentric hypertrophy with minimal concentric RV hypertrophy, severe RA enlargement, interventricular septal flattening in both diastole and systole (most profound in systole), severe tricuspid valve regurgitation, moderate-to-severe main PA enlargement, mild pulmonic valve insufficiency, and abnormal RV ejection profiles with mid-systolic notching in conjunction with a small, underfilled LV (Figures 1-4). A limitation of these subjective changes is that they lack information about the functional capacity and systolic function of the right heart.

In people, right ventricular ejection fraction (RVEF) is considered to be one of the most important measures of RV systolic function. Invasive cardiac right heart catheterization and cardiac magnetic resonance imaging are considered the gold standards for assessment of RV volumes, RVEF, and RV-PA coupling. Echocardiographically, alterations in RV ejection profiles (mid- and later-systolic notching) have also correlated with RV systolic function in human patients. This notching is from early arrival of reflected arterial waves from the noncompliant pulmonary vasculature. ${ }^{15}$ Tricuspid annular systolic plane excursion (TAPSE), a method used for evaluation of RV systolic function in people, has recently been characterized in dogs. ${ }^{16}$ This method has shown a reasonably good correlation with RVEF. ${ }^{17,18}$ Although not measured in this patient, in future patients, it would be interesting to compare qualitative right heart improvements to semiquantitative TAPSE.

RV mass decreased in people undergoing pulmonary endarterectomy to treat chronic thromboembolic PH. ${ }^{19}$ Sildenafil has also been shown to affect heart mass in $\mathrm{PH} .{ }^{20}$ Sildenafil is a highly selective phosphodiesterase-5 inhibitor that causes PA vasodilation by increasing vascular concentrations of cyclic guanosine monophosphate, resulting in nitric oxide-mediated vasodilation. Sildenafil decreases PA pressure and PVR. ${ }^{3,5}$ It may also have effects on pulmonary vascular remodeling. ${ }^{21,22}$ Two mechanisms have been proposed to explain its effects. The first is due to a decrease in PVR, resulting in a decrease in RV wall stress. The second mechanism involves blocking the intrinsic catabolism of cyclic guanosine monophosphate, suppressing chamber and cardiomyocyte hypertrophy. ${ }^{23}$ Investigators using sildenafil as treatment in experimentally induced $\mathrm{PH}$ in rats recently found that sildenafil prevented and partially reversed ultrastructural, molecular, and functional remodeling of failing RV myocytes. ${ }^{22}$ In this patient, causes of the profound improvement in clinical signs and structural right heart improvements remain speculative. It is possible that the inciting cause of the severe $\mathrm{PH}$ subsided or that the improvement was multifactorial, including resolution of the inciting cause, oxygen therapy, and sildenafil. Regardless of the cause of both the $\mathrm{PH}$ and the reasons for improvement, the remarkable echocardiographic improvements of the right heart are noteworthy.

\section{Conclusion}

To the authors' knowledge, significant echocardiographic improvement in right-sided cardiac pathology has not been reported in dogs with severe $\mathrm{PH}$. Echocardiography provides the most informative and least invasive method of evaluating cardiac structure and function in the veterinary clinical setting. The complex, asymmetric geometry of the right heart can make echocardiographic techniques more challenging, but they continue to prove a useful tool in the absence of invasive and expensive techniques such as cardiac catheterization and cardiac magnetic resonance imaging. Right heart changes associated with $\mathrm{PH}$ have traditionally been thought to be irreversible, but there is a recent trend toward more thorough assessment of the right heart and its utility in making diagnoses, prognoses, and monitoring treatment.

\section{Disclosure}

The authors report no conflicts of interest in this work.

\section{References}

1. Johnson L, Boon J, Orton EC. Clinical characteristics of 53 dogs with Doppler-derived evidence of pulmonary hypertension: 1992-1996. J Vet Intern Med. 1999;13(5):440-447.

2. Kellihan H, Stepien R. Pulmonary hypertension in dogs: diagnosis and therapy. Vet Clin North Am Small Anim Pract. 2010;40(4):623-641.

3. Bach JF, Rozanski EA, MacGregor J, Betkowski JM, Rush JE. Retrospective evaluation of sildenafil citrate as a therapy for pulmonary hypertension in dogs. J Vet Intern Med. 2006;20(5):1132-1135.

4. Forfia P, Roberts J. Diagnosis and assessment of pulmonary vascular disease by Doppler echocardiography. Pulm Circ. 2011;1(2):160.

5. Kellum HB, Stepien RL. Sildenafil citrate therapy in 22 dogs with pulmonary hypertension. J Vet Intern Med. 2007;21(6):1258-1264.

6. Kellihan H, Stepien RL. Pulmonary hypertension in canine degenerative mitral valve disease. J Vet Cardiol. 2012;14(1):149-164.

7. Stepien RL. Pulmonary arterial hypertension secondary to chronic left-sided cardiac dysfunction in dogs. J Small Anim Pract. 2009;50: 34-43.

8. Toyoshima Y, Kanemoto I, Arai S, Toyoshima H. A case of long-term sildenafil therapy in a young dog with pulmonary hypertension. $J$ Vet Med Sci. 2007;69(10):1073-1075.

9. Brown AJ, Davison E, Sleeper MM. Clinical efficacy of sildenafil in treatment of pulmonary arterial hypertension in dogs. J Vet Intern Med. 2010;24(4):850-854. 
10. McLaughlin VV, Presberg KW, Doyle RL, et al. Prognosis of pulmonary arterial hypertension: ACCP evidence-based clinical practice guidelines. Chest. 2004;126(Suppl 1):S78-S92.

11. Vitale CB, Gross TL, Magro CM. Vaccine-induced ischemic dermatopathy in the dog. Vet Dermatol. 1999;10(2):131-142.

12. Douglas WW, Tazelaar HD, Hartman TE, et al. Polymyositisdermatomyositis-associated interstitial lung disease. Am J Respir Crit Care Med. 2001;164(7):1182-1185.

13. Nagai S, Kitaichi M, Itoh H, Nishimura K, Izumi T, Colby TV. Idiopathic nonspecific interstitial pneumonia/fibrosis: comparison with idiopathic pulmonary fibrosis and BOOP. Eur Respir J. 1998;12(5): 1010-1019.

14. Bogaard HJ, Abe K, Vonk-Noordegraaf A, Voelkel NF. The right ventricle under pressure: cellular and molecular mechanisms of right-heart failure in pulmonary hypertension. Chest. 2009;135(3):794-804.

15. Arkles JS, Opotowsky AR, Ojeda J, et al. Shape of the right ventricular Doppler envelope predicts hemodynamics and right heart function in pulmonary hypertension. Am J Respir Crit Care Med. 2011;183(2): 268-276.

16. Pariaut R, Saelinger C, Strickland KN, Beaufrère H, Reynolds CA, Vila J. Tricuspid annular plane systolic excursion (TAPSE) in dogs: reference values and impact of pulmonary hypertension. J Vet Intern Med. 2012;26(5):1148-1154.
17. Sato T, Tsujino I, Ohira H, et al. Validation study on the accuracy of echocardiographic measurements of right ventricular systolic function in pulmonary hypertension. J Am Soc Echocardiogr. 2012;25(3):280-286.

18. Nijveldt R, Germans T, McCann GP, Beek AM, van Rossum AC. Semi-quantitative assessment of right ventricular function in comparison to a 3D volumetric approach: a cardiovascular magnetic resonance study. Eur Radiol. 2008;18(11):2399-2405.

19. Delcroix M, Vonk-NoordegraafA, Fadel E, Lang I, Simonneau G, Naeije R. Vascular and right ventricular remodelling in chronic thromboembolic pulmonary hypertension. Eur Respir J. 2012;41(1):224-232.

20. van Wolferen SA, Boonstra A, Marcus JT, et al. Right ventricular reverse remodelling after sildenafil in pulmonary arterial hypertension. Heart. 2006;92(12):1860-1861.

21. Schermuly RT, Kreisselmeier KP, Ghofrani HA, et al. Chronic sildenafil treatment inhibits monocrotaline-induced pulmonary hypertension in rats. Am J Respir Crit Care Med. 2004;169(1):39-45.

22. Xie YP, Chen B, Sanders P, et al. Sildenafil prevents and reverses transverse-tubule remodeling and $\mathrm{Ca}^{2+}$ handling dysfunction in right ventricle failure induced by pulmonary artery hypertension. Hypertension. 2012;59(2):355-362.

23. Takimoto E, Champion HC, Li M, et al. Chronic inhibition of cyclic GMP phosphodiesterase 5A prevents and reverses cardiac hypertrophy. Nat Med. 2005;11(2):214-222.
Veterinary Medicine: Research and Reports

\section{Publish your work in this journal}

Veterinary Medicine: Research and Reports is an international, peer-reviewed, open access journal publishing original research, case reports, editorials, reviews and commentaries on all areas of veterinary medicine. The manuscript management system is completely online and includes a very quick and fair peer-review system.

\section{Dovepress}

Visit http://www.dovepress.com/testimonials.php to read real quotes from published authors. 